\title{
Examining self-efficacy during learning: variability and relations to behavior, performance, and learning
}

\author{
Matthew L. Bernacki • Timothy J. Nokes-Malach • \\ Vincent Aleven
}

Received: 15 November 2013 / Accepted: 4 November 2014

(C) Springer Science+Business Media New York 2014

\begin{abstract}
Self-regulated learning (SRL) theorists propose that learners' motivations and cognitive and metacognitive processes interact dynamically during learning, yet researchers typically measure motivational constructs as stable factors. In this study, self-efficacy was assessed frequently to observe its variability during learning and how learners' efficacy related to their problem-solving performance and behavior. Students responded to self-efficacy prompts after every fourth problem of an algebra unit completed in an intelligent tutoring system. The software logged students' problem-solving behaviors and performance. The results of stability and change, path, and correlational analyses indicate that learners' feelings of efficacy varied reliably over the learning task. Their prior performance (i.e., accuracy) predicted subsequent self-efficacy judgments, but this relationship diminished over time as judgments were decreasingly informed by accuracy and increasingly informed by fluency. Controlling for prior achievement and self-efficacy, increases in efficacy during one problemsolving period predicted help-seeking behavior, performance, and learning in the next period. Findings suggest that self-efficacy varies during learning, that students consider multiple aspects of performance to inform their efficacy judgments, and that changes in efficacy influence self-regulated learning processes and outcomes.
\end{abstract}

Keywords Self-efficacy· Self-regulated learning · Intelligent tutoring systems · Problem-solving

When individuals are given a task and are asked to direct their own learning, self-regulated learning (SRL) theorists propose that what follows is a loosely sequenced process in which cognitive, metacognitive, and motivational components mutually influence outcomes and one another (Winne and Hadwin 1998; 2008; Zimmerman 2000a, b; Zimmerman and Schunk 2011). These theories generally propose that, learners define the task, set a goal, make a plan

M. L. Bernacki $(\bowtie)$

University of Nevada Las Vegas, 4505 South Maryland Pkwy \#3003, Las Vegas, NV 89154, USA

e-mail: matt.bernacki@unlv.edu

T. J. Nokes-Malach

University of Pittsburgh, Pittsburgh, PA, USA

V. Aleven

Carnegie Mellon University, Pittsburgh, PA, USA 
for achieving the goal, and carry out that plan using cognitive strategies. Learners metacognitively monitor the appropriateness of the learning goal, progress toward the goal, and the effectiveness of chosen strategies. They then exert metacognitive control by adapting a goal or the strategies used to achieve this goal if they determine their approach has not been successful.

Whereas the SRL process is at times described as a primarily metacognitive process (Winne and Hadwin 1998; Winne 2011), theorists also propose that learners must possess sufficient motivation to initiate and sustain their engagement (Winne and Hadwin 2008; Zimmerman 2011). When engaged in learning, the strategies learners employ and the monitoring decisions they make are further influenced by the types and levels of motivations they possess. It is important to note that self-regulated learning is understood to be an iterative, cyclical process. Within an iteration of the SRL process, motivation influences behavior and, in future iterations, motivation may be affected by past motivation, the consequences of a behavior, or products of monitoring processes. As a result, motivation is conceived of as a dynamic component of the SRL process that varies over the course of a learning task.

As an example, let us focus on the motivational construct of self-efficacy as students solve a set of math problems. Bandura (1986, 1997, 2006) describes self-efficacy judgments as being specific to a learning task and influenced by one's performance during the course of a task. Before students solve problems, they have an initial sense of their capability to successfully complete those problems. As they begin solving problems, they assess their performance in light of feedback, and experience increased efficacy after successful attempts or decreased efficacy after unsuccessful attempts. To the degree that feelings of selfefficacy predict future behaviors, we should expect students to behave differently when their efficacy is high versus when it is low.

Considerable research has examined the role motivation plays in self-regulated learning, and prior studies have found that self-efficacy predicts learners' cognitive and metacognitive behaviors (Pajares 2008). However, much of this work has treated motivational constructs as stable components of the SRL process that do not vary during learning; researchers typically assess motivation only at the outset or completion of a task. This treatment of motivation conflicts with models of self-regulated learning and, in the case of self-efficacy, with the theoretical assumptions about the construct itself.

In this paper, we examine self-efficacy as a motivational construct that is theorized to operate in a dynamic fashion during learning. To achieve this, we employed a microgenetic methodology commonly used in cognitive developmental psychology. Microgenetic studies are conducted when researchers anticipate extensive change in a phenomena and accordingly 1) observe the entire span in which the change is anticipated to occur, 2) conduct dense observations of the phenomenon until changes give way to stability, and 3) examine multiple trials so that quantitative and qualitative differences in the phenomena might be observed (Siegler and Crowley 1991). In this case, we observed students' development of math skills over an entire math unit (i.e., the learning task), and observed their behavior during all problem solving attempts in the unit. In addition to the observations of behavior and performance that are common to the classic microgenetic method, we incorporated a microgenetic self-report method (Bernacki et al. 2013) that involves frequent, automated prompts to elicit self-efficacy judgments from learners as they solve a series of math problems. In this way, we extend the microgenetic approach for use with motivational phenomena and examined responses to prompts alongside logs of students' learning behaviors during problem attempts as captured by the intelligent tutoring system (ITS). This prompted self-report method is somewhat similar to microanalytic approaches previously used to examine self-regulated learning processes (Cleary 2011; Cleary and Zimmerman 2001). However, our approach is unique in that the prompts were embedded 
in an automated fashion within the learning task so that we could observe sequential learning events at a fine grain of detail and examine how changes in efficacy and learning events relate to one another. We used this method to test specific assumptions about the dynamic nature of selfefficacy during learning tasks, sources of efficacy judgments, and the implication of self-efficacy for learning.

Before describing this approach in greater detail, we first summarize the theorized role of motivation in the SRL process with an explicit focus on the role of self-efficacy. We then contrast the variety of measurement approaches self-regulated learning researchers have employed to investigate relationships between SRL processes, and describe the unique advantages that the combination of frequent self-reports and log-file analysis offers when investigating complex relations between motivation and learning during a task.

\section{The dynamic role of self-efficacy in learning}

Consistent amongst theories that highlight motivation as a critical component of SRL (Efklides 2011; Pintrich 2000; Winne and Hadwin 2008; Zimmerman 2000a, b, 2011) is an emphasis on the role of self-efficacy during self-regulation. Self-efficacy refers to the belief in one's capability to perform at a particular level on a task (Bandura 1994). Self-efficacy is conceptualized as a context-specific phenomenon (Bandura 1997, 2006) that has been shown to be "sensitive to subtle changes in students' performance context, to interact with self-regulated learning processes, and to mediate students' academic achievement" (Zimmerman 2000b, p. 82). Drawing on Bandura's conceptualization in social cognitive theory, self-regulated learning theories describe self-efficacy as a dynamic component within the SRL process; self-efficacy is influenced by learners' prior behavior and influences their future behaviors. Zimmerman (2000a, b, 2011) has long held that self-efficacy plays an essential role in a social cognitive model of self-regulation. During the forethought phase, individuals' feelings of self-efficacy influence the proximal goals they set in future iterations of the SRL cycle. During later performance and self-reflection phases, efficacy beliefs influence individuals' metacognitive judgments about the learning strategies they have employed.

In their COPES model, Winne and Hadwin (2008) describe self-efficacy beliefs as one of multiple conditions that influence how learners define a learning task. This task definition process influences the goals learners will set and ultimately, the type of cognitive operations (i.e., learning tactics) they will employ to try and satisfy their goals. For example, if a learner possesses a high level of efficacy for a specific problem-solving task, she may set a goal to complete a problem without any assistance. The resulting operation would be persistence through all the steps in the upcoming problem without requesting any hints from the software. Self-efficacy beliefs are themselves also a motivational product that arises from the learning process. In the example, if the learner was able to solve the problem without assistance, her self-efficacy may increase. If she could not, it may decrease.

\section{Research on self-efficacy and relation to learning}

Efficacious learners tend to be more willing to engage in a task (Bandura and Schunk 1981; Pajares and Miller 1994), set challenging goals, and maintain strong commitments to achieving their goal (Pajares 2008). Self-efficacy has also been found to influence performance during learning tasks (Bandura 1997; Hackett and Betz 1989; Lent et al. 1984: Shell and Husman 2008). With respect to metacognitive processes, learners who have high self-efficacy for a 
learning task tend to engage in increased levels of metacognitive monitoring of both their understanding of task content and the relevance of learning materials (Moos 2014).

Whereas each of these studies produced important findings regarding relations between self-efficacy and learning, each also employed assessment practices that possess a limited ability to capture variability in learner's self-efficacy or learning behaviors. Because selfefficacy was assessed at a single time point prior to the learning task, no changes in selfefficacy could be detected. As a result, all actions are associated with students' initial selfefficacy for the task, and could not be associated with increases or decreases in efficacy over the course of learning, or with a momentary level of efficacy the student experienced when such monitoring was being conducted. With a handful of exceptions (Cleary and Zimmerman 2001; McQuiggan et al. 2008), it is uncommon for researchers to collect sufficient data points that variation in these constructs can be analyzed (i.e., one time point cannot accommodate considerations of linear change; pre-post assessments cannot accommodate non-monotonic change). In this study, we examine self-efficacy frequently during learning so that we can better understand not only how initial efficacy predicts self-regulated learning behaviors, but also how changes in efficacy can predict behavior, and how efficacy can change as a result of behavior.

\section{Self efficacy as a dynamic component of SRL}

Because our research hypotheses are predicated on the assumption that learners' level of selfefficacy changes over a learning task, we first must confirm that there is sufficient change in efficacy over our observations to warrant further investigation. To establish this, we adopted a set of analyses used by personality researchers to detect stability and change in psychological constructs (Caspi, Shiner \& Roberts, 2005). These analyses include assessments of differential continuity (i.e., degree of correlation between an individual's reports over multiple observations), mean-level change (i.e., differences in the mean-level of efficacy for the sample across observations), and individual-level change (i.e., percent of individuals who report a change in efficacy that exceeds a level associated with measurement error). To date, these methods have been used to examine motivational constructs like achievement goals over a semester of classroom learning (Fryer and Elliot 2007; Muis and Edwards 2009) and over a series of technology-enhanced learning tasks (Bernacki et al. 2014). These types of analyses confirm that motivation varies across learning tasks (i.e., psychology exams and assignments; math units), but they have yet to be conducted with observations of motivation within a single learning task. If variability is observed, then conducting multiple observations during a single learning task enables us to explore research questions that test assumptions about the concurrent, dynamic relations amongst motivational, metacognitive, and cognitive processes proposed by theories of self-regulated learning.

\section{Design choices in self-regulated learning research}

A persistent topic in the study of self-regulated learning is the paradoxical decision one must make regarding design choices. Because self-regulated learning is a complex phenomenon composed of many subprocesses, researchers have often chosen to either investigate the multifaceted process at a general level, or focus in on a specific set of subprocesses as they unfold during a single period of learning under close observation. Studies employing general approaches typically utilize survey methods (e.g., Motivated Strategies for 
Learning Questionnaire; Pintrich et al. 1991) and are particularly useful for addressing questions about learners' aptitude to self-regulate (Winne and Perry 2000) - the relationships among broadly defined SRL processes - and relations to learning outcomes. However, findings typically lack specificity with respect to the amount and quality with which students engage in processes like monitoring or strategy use, or the timing and sequencing of those processes. At the other end of this continuum are studies that select a small set of SRL processes and examine them closely in the context of a single learning task. These types of studies usually capture SRL events (Winne and Perry 2000) using process-oriented methodological approaches like microanalytic methods (Cleary 2011), think aloud protocols (Greene et al. 2011), and trace methodologies (Aleven et al. 2010; Azevedo et al. 2013) to observe individual SRL processes as they unfold during learning.

As self-regulated theories become more explicit in their descriptions of the relations between components of SRL models and environmental factors (i.e., Winne and Hadwin 2008; Zimmerman 2011) researchers can now examine theoretical assumptions regarding relations amongst subprocesses of SRL models. In order to test assumptions about cyclical and mutually influential processes like those proposed in SRL theories, it becomes increasingly important for researchers to capture evidence of these SRL events as they unfold during learning and to conduct analyses that investigate multiple relationships simultaneously.

To date, the most common methodological answer to this dilemma has been to conduct think aloud protocols to observe self-regulated learning processes as they unfold (Ericsson and Simon 1980; Greene et al. 2011). Numerous studies by Azevedo, Greene, Moos and colleagues (e.g. Azevedo 2005; Greene and Azevedo 2009; Moos 2014) have used think aloud protocols to examine learning as it occurs over a single task spanning a 20-40 minute period and to identify the effect that SRL subprocesses have on learning outcomes. In these studies, learners self-report their self-regulatory processes, which are then categorized as events reflecting planning, monitoring, strategy use, environmental structuring, and interest (Greene and Azevedo 2007; 2009). Think aloud protocols provide an opportunity to examine sequenced processes and examine contingent behaviors and processes. Examining the frequency with which SRL processes are reported (e.g., Azevedo et al. 2010), it can be inferred that learners tend to report many instances of metacognitive processes (e.g., positive and negative judgments of learning, feelings of knowing) and strategy use (e.g., reading, re-reading, use of a mnemonic) but relatively few instances of experiencing a motivational state. This may be due in part to think aloud protocols' reliance on the learner to initiate the reporting of cognitive, metacognitive, and motivational processes. Because learners infrequently report on their motivation when observed using this approach, and because no research has been conducted that examines think aloud data for evidence of motivational states besides interest in the task, it remains an open question as to how learners' motivation varies over the course of learning, and what role it plays in the broader learning process. To address this research question, researchers must employ a methodology that intentionally elicits reports of motivation from individuals during learning.

For example, one way to examine how a particular type of motivation relates to other SRL processes, is to prompt explicit self-reports of that motivational construct, and to do so at intervals that a) match the assumptions about the stability of the construct and b) support the intended analysis. Cleary's (2011) microanalytic approach to assessing self-regulated learning draws on the microanalytic tradition begun by Bandura (e.g., Bandura et al. 1982) and offers a potential solution to this methodological design challenge. SRL microanalysis involves close observation and detailed recording of behavioral processes, as well as timely questioning about less overt processes. For instance, Cleary and Zimmerman (2001) examined individuals' selfefficacy for free throw shooting by periodically asking participants "How sure are you that you 
can make the next free-throw?" before and after a series of made and missed attempts. This kind of repeated prompting enabled them to test hypotheses about relations between selfefficacy and attributions, and could also be used to examine how a motivational construct like self-efficacy varied across attempts. These self-efficacy reports could also be situated within the sequence of SRL processes to examine how the events that precede a prompt predict selfefficacy reports, and how feelings of efficacy predict future events. This approach is quite informative, but also requires extensive effort to collect data. Participants must be observed individually and researchers must actively prompt participants during the task. Data must then be transcribed, and coordinated across different modalities (i.e., survey instruments, written logs, etc.) before analyses can ensue. Ultimately, this limits the number of participants one can observe, which can in turn limit the statistical power available to conduct complex analyses with many variables.

\section{The present study}

In this study, we examine whether motivational constructs such as self-efficacy truly operate dynamically during learning, and how changes in efficacy relate to past and future learning events. To accomplish this, we embed self-efficacy prompts within a unit of Cognitive Tutor Algebra, an intelligent tutoring system that adolescents used to learn algebra. As they use the software to learn, it recorded their actions, which can be used as inferential evidence of selfregulated learning processes. This measurement approach draws upon features of the microanalytic approach (Bandura 1997; Cleary 2011) to simultaneously observe motivational, cognitive, and metacognitive processes in a single medium. The fully automated approach we employ logs students' learning processes in sequence and prompts students to periodically self-assess their degree of efficacy, producing a fine-grained record of behavior and a systematically sampled stream of motivational data. This allows for the collection of detailed behavioral and motivational data from considerably larger samples of learners than can be collected with other methods and supports the testing of models that approximate the dynamic relations between motivation and learning processes proposed in SRL theory.

In the Cognitive Tutor, students receive correctness feedback and can observe their progress towards learning goals. When given these forms of feedback, students are likely to adjust their perceptions of their own skillfulness (i.e., mastery experience; Bandura 1997; Usher and Pajares 2006), which in turn may affect their future approach to learning. We anticipate that, when learning with a software like the Cognitive Tutor that provides opportunities to solve problems, consider feedback, and self-regulate learning, learners will adjust their efficacy over a series of successful and unsuccessful problem solving attempts and adjust how they regulate their learning in light of the efficacy they feel for the task.

We focused our observations in this study on learners' self-efficacy for math problem solving and examined how efficacy changed over time and related to students' prior and subsequent learning processes. Prior theory suggests that individuals' feelings of efficacy are 1) determined by past achievement experiences, 2) interactive with self-regulated learning processes and 3) predictive of students' performance on academic tasks. Past research has treated self-efficacy as a stable motivational phenomenon and measured it once per task, despite theoretical assumptions that the efficacy that learners feel is sensitive to their performance context and recent experiences (Bandura 1997; Zimmerman 2000a, b; Zimmerman and Schunk 2008; Zimmerman 2011). Because students' self-efficacy judgments are conceptualized in theory to be context-specific judgments that change over the course of a learning 
task and interact with learning in a reciprocally deterministic fashion, we propose that it is more appropriate to examine self-efficacy repeatedly during learning to capture change and to examine potential "mediating effects" on academic achievement described by (Zimmerman 2000b). This approach has yet to be taken and can help clarify whether self-efficacy does indeed vary over a learning task and whether the role it plays in learning is as dynamic as is proposed in SRL theories.

\section{Research questions and hypotheses}

As a preliminary research question, we aimed to confirm the sensitivity of self-efficacy judgments to events that occur during learning (per Bandura 1986, 1997) using stability and change methodologies (Caspi et al. 2005). We questioned 1) whether self-efficacy would vary during a math unit and hypothesized that student's self-reported self-efficacy should vary over the course of the learning task. Once it could be established that selfefficacy varies during a learning task, we tested two research questions stemming from self-efficacy theory: 2) whether judgments of efficacy were informed by prior performance and 3) whether efficacy judgments would predict future performance. Because self-regulated learning theorists propose that changes in self-efficacy are induced by prior SRL events and have implications for future events (Winne and Hadwin 2008; Zimmerman 2011), we conducted an additional pair of analyses to explore 4) how prior and concurrent performance metrics relate to changes in self-efficacy during learning and 5) how changes in students' self-efficacy judgments in one period predicted their selfregulated learning behaviors, performance, and learning when solving future math problems.

\section{Methods}

Participants

Participants included 107 9th grade students drawn from regular tracks of algebra classes at a single high school in the Mid-Atlantic United States. The sample was $56 \%$ male and $98 \%$ of students were identified by the school district as Caucasian. Twenty four percent of the students received free or reduced price lunch, and $15.5 \%$ had a special education designation. All participants were enrolled in algebra courses that utilized Cognitive Tutor Algebra (CTA; Carnegie Learning 2011) as a supplement to their classroom algebra lessons. Students completed CTA units at their own pace on laptops provided to students during two classes per week. The participants in this study were all those who completed the sixth unit in the CTA curriculum (on linear equation solving) and responded to periodic items prompting self-reports of motivation.

\section{Measures}

We assessed students' self-efficacy for solving math problems in the linear equations unit (i.e., Unit 6) using an automated prompt embedded in the Cognitive Tutor software and assessed their learning behaviors using a log-file generated by software. We first describe the Cognitive Tutor Algebra environment, and then detail the design of the tool used to assess self-efficacy for math problems. 
Cognitive Tutor Algebra Cognitive tutors are a family of intelligent tutoring systems (c.f. Koedinger and Aleven 2007) that combine the disciplines of cognitive psychology and artificial intelligence to construct computational cognitive models of learners' knowledge (Koedinger and Corbett 2006). Cognitive tutors monitor students' performance and learning via model tracing and knowledge tracing. That is, the Cognitive Tutor runs step-by-step through a hypothetical cognitive model (representing the current state of the learner's knowledge) as the learner progresses through a unit. This allows the tutor to provide real-time feedback and context-specific advice on problem steps. In Cognitive Tutor Algebra, learning is defined as the acquisition of knowledge components, or skills that are targeted in a specific unit. Learners complete a series of units that aim to develop a specific set of algebra skills. Each unit provides students with an introductory text summarizing a topic, some worked examples to demonstrate key concepts, and then a problem set. During problem solving, students receive step-by-step guidance and feedback from the tutor, supplemented with tools students can use to self-regulate their learning. These tools include a hint button that provides context-specific hints, a glossary of terms relevant to the content and, at times, a worked example of a problem similar to those they are to complete in the unit. Students also have the ability to monitor their skill development by clicking on the skillometer (Long and Aleven 2013), which displays the skills targeted in the unit and students current skill rating. This rating is determined by student' performance on prior problem steps testing these skills. A screen shot of the Cognitive Tutor interface for problem solving tasks appears in Fig. 1. In this study, we observed algebra students' learning behavior as they completed a single unit in the Cognitive Tutor that targeted skills associated with solving problems involving linear equations and consider these behaviors as potential evidence of students' self-regulation of their learning.

Tracing SRL in log-files Students interact with the Cognitive Tutor by answering problem steps, requesting hints, or accessing resources. Each of these transactions is logged in sequence, along with a reference to the problem step and the time at which it occurred. When examining the series of transactions associated with a single problem step, it is possible

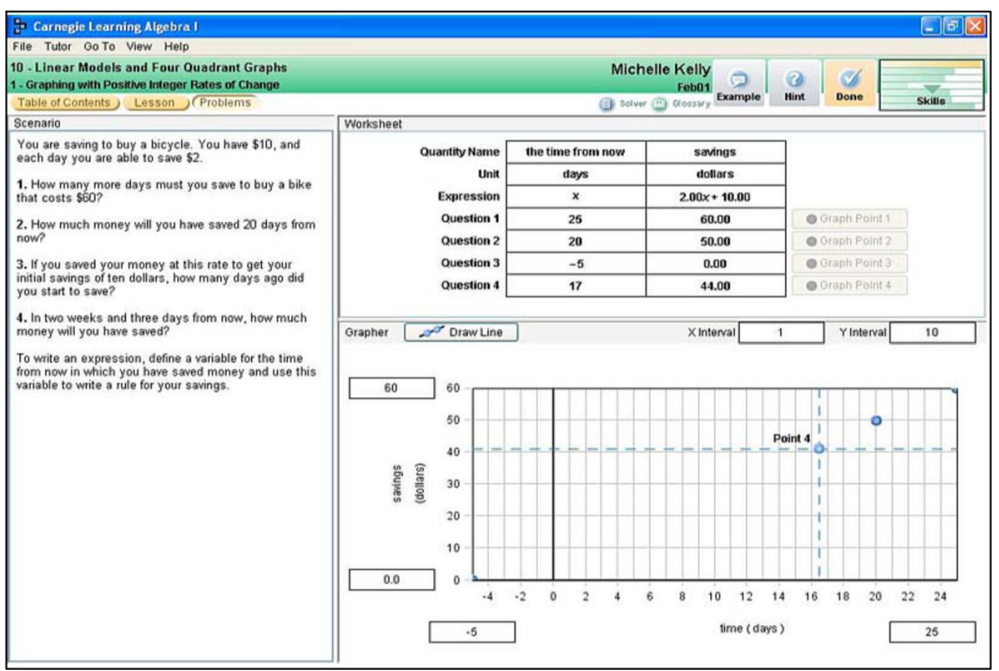

Fig. 1 Screen shot of Cognitive Tutor Algebra 
to hypothesize students' self-regulatory processes. For example, when first presented with a problem step (i.e., Fig. 1; white boxes with bold type), the learner could consider the step and decide whether she can complete it accurately. This process involves a metacognitive monitoring process in which the learner decides whether she possesses sufficient understanding to attempt to solve the problem step (a metacognitive control strategy; Winne and Hadwin 1998) or whether she should request a hint (an alternate strategy). Upon submission of an answer, the learner receives immediate feedback about the correctness of the attempt, which in turn can be considered alongside the problem statement. If correct, the learner can attribute her successful completion of the step to the possession of knowledge and has the opportunity to increase her perception of efficacy for the problem type accordingly. If incorrect, she can attribute the error to a lack of knowledge and then either request a hint or reconsider the problem step and answer again. To that end, each transaction with the Cognitive Tutor is composed of both a metacognitive monitoring process (which may not necessarily be explicit or conscious) and a control process that is logged in the form of a correct, incorrect, or hint attempt. Aleven et al. (2006) have proposed a formal model which catalogs the metacognitive monitoring and control processes involved when learners make a decision to venture answers or seek help. While their metacognitive model of help seeking does not align explicitly to a single theory of SRL, the corresponding sequences of transactions in a tutor log can be thought of as traces of SRL cycles (i.e., metacognitive monitoring and control events per Winne and Hadwin 1998) within a problem.

It is important to note that the SRL processes described are hypothesized; they may occur at times and not at other times, and they may occur more frequently with some learners than with others. For example, the decision to seek help may not always be the result of metacognitive monitoring and control (e.g., it may be simply a quick reaction to feedback indicating an error). Likewise, attributions of success/failure may not accurately take into account the amount of help received from the tutoring system. As with all interpretation of behavioral data, tracing cognitive and metacognitive processes from such behaviors is an inferential pursuit and findings should be interpreted accordingly.

Self-efficacy prompts In the unit under analysis (i.e., Unit 6, Linear Equations), we implemented a "pop-up" tool that was created by the software provider to assess student perceptions about features of the math problems they encounter in Cognitive Tutor Units. When this tool is enabled, students complete all the steps in a problem, click the "Done" button, and are then presented with a single item that pertained to their problem solving experiences. In this unit, a set of four items was repeatedly presented in a fixed sequence (i.e., one item after each completed problem) so that each construct was sampled after every fourth problem. This study considers students' responses to an item administered after every fourth problem, which prompted students to report their level of efficacy for completing similar math problems in the future (i.e., "How confident are you that you could solve a math question like this one in the future?"). Students rated their level of efficacy by selecting a value on a 10-point Likert scale ranging from 1 (not at all confident) to 10 (completely confident). Table 1 shows the mean, deviation and range in efficacy scores for the group in response to each efficacy prompt. A single-item approach has been employed in prior studies of self-regulation during athletic practice (Cleary and Zimmerman 2001) and by Ainley and Patrick (2006). As documented by Ainley and Patrick (2006), the use of a single item to observe a psychological construct is generally considered to be valid when the construct is unambiguous to the respondent. Because students were simply asked to indicate their confidence that they could perform 
Table 1 Descriptive statistics for self-efficacy reports for math problems $(N=107)$

\begin{tabular}{llll}
\hline & $M$ & SD & Range \\
\hline Self-efficacy prompt 1 & 7.93 & 2.41 & 9 \\
Self-efficacy prompt 2 & 8.15 & 2.27 & 9 \\
Self-efficacy prompt 3 & 7.90 & 2.47 & 9 \\
Self-efficacy prompt 4 & 7.77 & 2.58 & 9 \\
\hline
\end{tabular}

an unambiguous task (i.e., "solve a math question like this one"), our approach meets this requirement.

\section{Procedure}

Students began completing Cognitive Tutor units in the second month of the school year and continued until the school year ended. Most students completed the unit under analysis in the fall semester, though those who proceeded at a slower pace completed the unit some time in the spring. After the school year was complete, we obtained log-files from the software provider that contained all of students' transactions with the software. This included their attempts at problem steps, use of tools available in the unit, and their responses to prompts. To assess students' learning processes, we segmented these logs of students' behavior into periods containing four problems, which occurred prior to the first efficacy prompt (i.e., Period 0 prior to Prompt 1), after the first efficacy prompt and prior to the second (i.e., Period 1, containing the next 4 problems after Prompt 1 was presented) and so on. The sequence of problem periods and efficacy prompts can be seen in Fig. 2.

We first examined responses to the four self-efficacy prompts to determine whether students reported variable levels of self-efficacy over the course of the task (RQ1). We then employed self-efficacy reports and summaries of learning processes in each period to test a statistical model that examined how performance and judgments that preceded a self-report prompt predicted self-efficacy ratings (RQ2) and how self-efficacy ratings predicted performance in subsequent solving periods (RQ3). We next conducted additional analyses to examine how changes in self-efficacy related to different aspects of prior performance (RQ4) and influenced future learning behaviors, performances, and outcomes (RQ5).

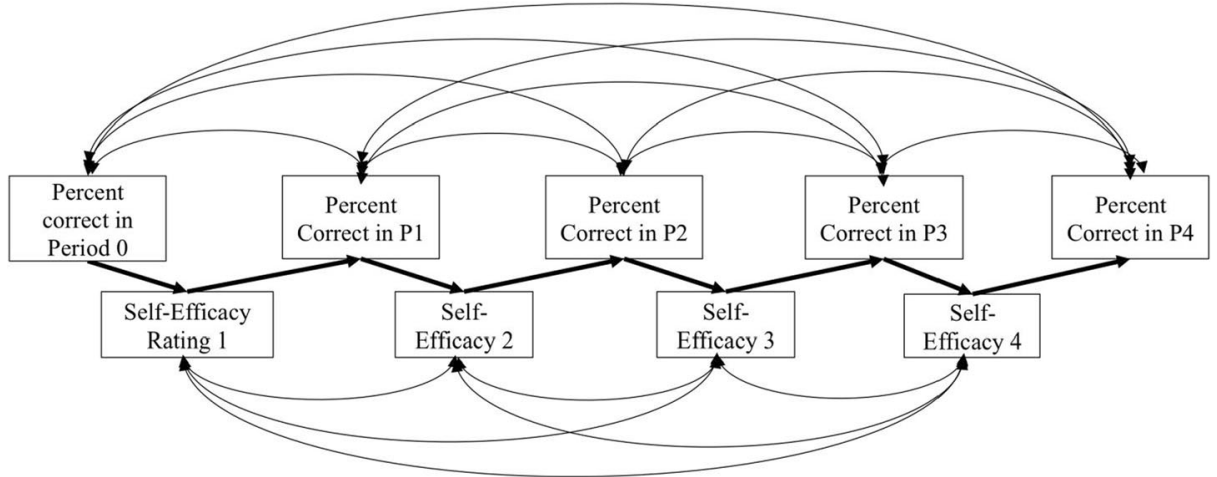

Fig. 2 Hypothesized path model 


\section{Results}

Research question 1: does self-efficacy vary reliably across problems?

We examined variation in self-efficacy for math problems with a common set of analyses used to assess stability and change in psychological constructs (Caspi et al. 2005; Muis and Edwards 2009; Fryer and Elliot 2007). These include examinations of differential continuity in the construct, mean-level change over time, and an analysis of reliable change within individuals over time.

Differential continuity To assess differential continuity in self-efficacy scores, we conducted a correlational analysis examining associations between all pairs of scores across the four observations. This analysis is similar to test-retest analyses for which correlations in excess of $r=.85$ suggest reliability in a construct across observations (Rosenthal and Rosnow 1991). The results are summarized in Table 2. Correlations between self-efficacy judgments ranged from $r(107)=.61$ to .86 across comparisons, suggesting a moderate to high level of continuity in efficacy across observations within a learner. While strongly correlated, efficacy judgments mostly failed to reach the threshold for test-retest reliability in settings where only measurement error is expected (i.e., $r=.85$; Rosenthal and Rosnow 1991). This suggests that the difference between correlation coefficients and this threshold values is indicative of intraindividual change across efficacy judgments made every few minutes during the problemsolving task.

Mean-level change To assess mean-level change, we conducted a repeated measures analysis of variance and tested whether we would find a significant linear, quadratic, or cubic function in self-efficacy scores across time. Means and standard deviations per prompt appear in Table 1. No significant linear, quadratic, or cubic function was found, $F \mathrm{~s}<2.90, P S=\mathrm{ns}$. We conclude that at the level of the sample mean, self-efficacy scores did not differ from one time point to the next. As noted in prior analyses of stability and change, non-significant levels of mean-level change can be obtained due to a lack of variability at the individual level, or to variability that includes equal amounts of increase and decrease which offset and result in a net change equivalent to zero, which masks variability within individuals' scores (Fryer and Elliot 2007; Muis and Edwards 2009). We explored individual level change to make this determination.

Individual-level change We examined changes in individuals' self-efficacy using a reliable change index (RCI; Christensen and Mendoza 1986; Jacobson and Truax 1991), which was developed to enable researchers who observe variability in a construct over multiple observations to distinguish between instances where variability can be attributed to imperfect

Table 2 Correlation matrix of self-efficacy scores across four observations

\begin{tabular}{lllll}
\hline & & 1 & 2 & 3 \\
\hline 1. & - & & \\
\hline 2. & Self Efficacy prompt 1 & $.74^{*}$ & - & \\
3. & Self Efficacy prompt 2 & $.71^{*}$ & $.86^{*}$ & - \\
4. & Self Efficacy prompt 3 & $.61^{*}$ & $.80^{*}$ & $.85^{*}$ \\
\hline
\end{tabular}

$* p<.05$ 
measurement by an instrument and reliable change representing actual changes in a construct between observations. To make this distinction, differences in scores are examined in contrast to the standard error of the difference score (i.e., the spread of the distribution of change scores to be expected if no change occurred). By comparing this value against a critical value set a priori (i.e., \pm 1.96 ), the RCI score can be used to categorize individuals as demonstrating a significant increase (i.e., RCI value $>1.96$ ), a significant decrease (i.e., $\mathrm{RCI}<-1.96$ ), or no significant change across a pair of observations (i.e., between \pm 1.96 ). Scores falling outside the range of \pm 1.96 are unlikely to result from measurement noise and are thus considered indicative of reliable change.

RCI scores are summarized in Table 3, which indicates the percentage of students who reported a statistically reliable increase or decrease (or no statistically reliable change) in their self-efficacy from one prompt to the next. Across the sample of 107 learners and all four observations, roughly half of learners reported a reliable change in their efficacy for math problems involving linear equations from one observation to another. Sixty percent of learners' reported at least one reliable change in self-efficacy over the duration of the task, and $38 \%$ showed two or three reliable changes. Because the degree of reliable change observed across self-efficacy judgments is similar to prior studies acknowledging intra-individual change in motivational variables (Bernacki 2014; Fryer and Elliot 2007; Muis and Edwards 2009), we conclude that our findings indicate that learners' self-efficacy varied reliably over observations within a single learning task. Thus we continued with analyses examining the sources that may predict changes in efficacy and whether efficacy judgments predicted future learning processes or outcomes.

Research questions $2 \& 3$ : How do learners' performances relate to future self-efficacy reports? And do these reports predict future performance?

Bandura (1997) theorized that prior performances are an important source informing selfefficacy judgments, and that self-efficacy is an important predictor of future performance. We tested these assumptions within the learning task using the path model that appears in Fig. 2. After segmenting data into periods of problem-solving transactions occurring between prompts, we tested whether performance (i.e., percent correct on all attempts at problem steps) in one period predicted the self-efficacy rating made immediately after the period (i.e., mastery experience predicts efficacy) and whether efficacy predicted performance in subsequent periods of problem solving.

Table 3 Reliable change index documenting changes in self-efficacy across 4 observations

\begin{tabular}{llll}
\hline & \multicolumn{2}{l}{ Change from self-efficacy } & \\
\cline { 2 - 4 } & Prompt 1 to 2 & Prompt 2 to 3 & Prompt 3 to 4 \\
\hline Reliable increase & $24 \%$ & $23 \%$ & $22 \%$ \\
No change & $60 \%$ & $51 \%$ & $49 \%$ \\
Reliable decrease & $16 \%$ & $26 \%$ & $29 \%$ \\
Percent exhibiting change & $40 \%$ & $49 \%$ & $51 \%$ \\
\hline
\end{tabular}

Percentages in "reliable increase" and "decrease reflect" the portion of the sample whose scores reflect a difference greater than one standard error of measurement, thus indicating true change 
Results indicate that, initially, students' performance in a prior period predicted their efficacy for the period immediately following, but also that the strength of this predicted effect declined over time (Fig. 3). As the predictive effect of past performance declined, associations between prior efficacy-levels and current efficacy judgments strengthened. This suggests that the criteria used to judge efficacy shifted gradually from reflection on past performance to a greater reliance on past feelings of efficacy. We also examined additional sources of efficacy judgments in analyses addressing Research Question 4.

As can be seen in Fig. 3, self-efficacy ratings were not significant predictors of performance in the period that immediately follows an efficacy rating. Contrary to theory (Bandura 1997), we did not obtain evidence that self-efficacy predicted future problem-solving performance in an ITS setting. We conduct additional analyses related to this theoretical assumption when addressing Research Question 5.

Research question 4: How do aspects of prior performance relate to changes in self-efficacy ratings?

Because students are known to consider multiple sources of information when rendering efficacy judgments, we examined which aspects of prior performance may have served as sources to inform efficacy judgments made during problem-solving. We first examined whether prior performance metrics including accuracy (i.e., percent of problem steps answered correctly in the previous set of 4 problems), improvements in accuracy (i.e., accuracy in the most recent period minus accuracy in the prior period), fluency (i.e., the speed with which subjects completed attempts at steps during the period), and improvements in fluency (i.e., speed of step completion in the most recent period compared to the previous period) were associated with changes in self-efficacy while completing a set of four math problems. Results of these correlational analyses appear in Table 4.

Across three periods of observation, recent improvements in performance and speed of task completion were both associated with subsequent increases in self-efficacy. The strength of association between accuracy metrics and change in self-efficacy decreased over the course of the learning episode, while the strength of the association between fluency metrics and change in self-efficacy increased. Taken together, these patterns suggest a shift in the heuristics that individuals use to judge their efficacy for a learning task. One interpretation of these findings is that, initially, self-efficacy judgments were predicated on accurate prior performances on a task, or with a performance heuristic. As individuals' performance improved over time, they began to focus on improvements in

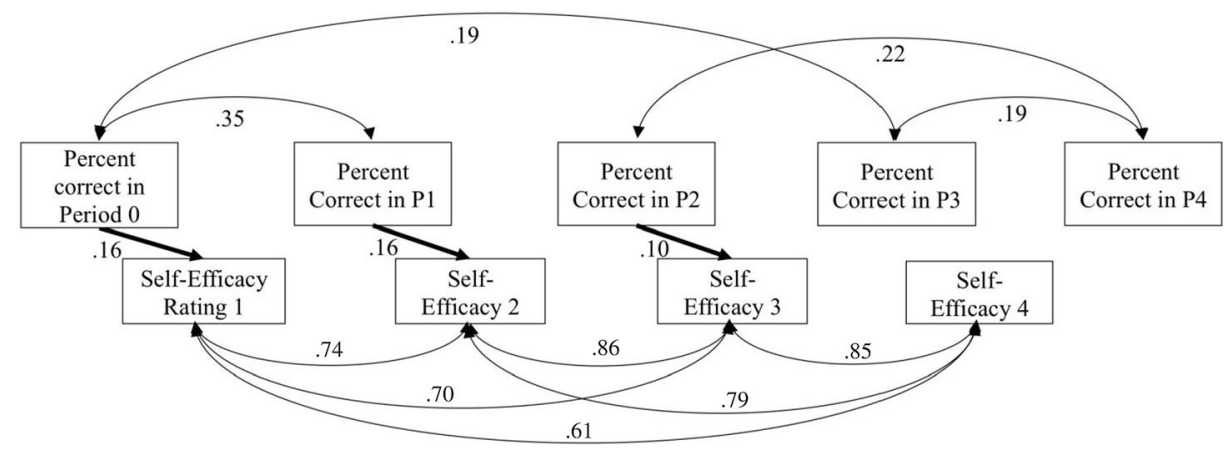

Fig. 3 Standardized solution to the path model $\chi 2(15)=15.07, p=0.44, C F I=1.00$, RMSEA 0.007, SRMR $=0.032$ 
Table 4 Correlations between prior and current performance metrics and increases in self-efficacy for math problems

Change in efficacy Period $2 \quad$ Period 3

during period 1

\begin{tabular}{llll}
\hline Accuracy in current period & .17 & .17 & .10 \\
Increase in Accuracy (compared to prior period) & $.26^{*}$ & $.30^{*}$ & .18 \\
Fluency in current period (seconds per problem step) & -.13 & $-.23^{*}$ & $-.51^{*}$ \\
Increase in Fluency (compared to prior period) & -.04 & -.14 & $-.53^{*}$ \\
\hline
\end{tabular}

$* p<.05$

Note. Negative relations between fluency and efficacy indicate an increase in efficacy as time spent on problem steps decrease

fluency, or the speed with which they could achieve accurate performances. As individuals consistently answer problem steps correctly (i.e., variation in accuracy diminishes), they increasingly rely on speed of completion as a fluency heuristic to continue updating their judgments of self-efficacy. We propose a possible reason for this shift in the "Discussion" section.

Research question 5: how do changes in efficacy relate to subsequent behavior, performance, and learning?

To determine how changes in efficacy influence future learning, we next conducted a series of partial correlations that examined relations between changes in self-efficacy over one set of four math problems and students' behavior, performance, and learning during the subsequent set of four problems. For these analyses, we focused on increases in efficacy experienced during the third period of problem solving (i.e., change from Self-Efficacy Prompt 3 to 4) and learning processes that occurred during the subsequent problem solving period (i.e., Period 4), while also controlling for prior performance (which is known to predict future performance; see Fig. 2) and prior levels of efficacy (which determine a baseline from which changes in efficacy judgments are made). We chose this period because, in our analysis of individual-level change, the greatest number of individuals indicated a reliable change in self-efficacy during this period (i.e., $51 \%$ of learners). We processed the log of students' behaviors to produce a set of measures indicating performance, learning, and self-regulated learning behavior in a Cognitive Tutor unit. These included Accuracy (i.e., percent of problem steps answered correctly in Period 4), Change in Accuracy (i.e., from Period 3 to Period 4), the total number of Hints requested by learners during problem steps and the Change in hints requested from the current period of problems (Period 3) to the next. A summary of the partial correlations appears in Table 5.

Controlling for prior performance and initial efficacy, students who experienced increases in self-efficacy during Period 3 requested significantly fewer hints when solving problems in Period 4 and decreased their tendency to seek help compared to their rate in the prior problemsolving period (Period 3). Collectively, these findings suggest that increases in efficacy were associated with less frequent help seeking during concurrent and future problem-solving periods. Increases in self-efficacy were also associated with higher accuracy in the subsequent unit and an improvement in accuracy in the future period. These findings suggest that increases in feelings of efficacy are associated with better performance and learning even after controlling for associations with prior performance and efficacy. 
Table 5 Partial correlation between changes in efficacy (Period 3 to 4) and learning measures

$d f=103 ;$ covariates $=$ Period $3 \%$ correct, self-efficacy prompt 3 score

\begin{tabular}{lcc}
\hline & $R$ & $p$ \\
\hline Learning behaviors & & \\
$\quad$ Total number of hints requested (Period 4) & -.32 & $<.01$ \\
$\quad$ Total hints requested from Period 3 to 4 & -.36 & $<.01$ \\
Performance & & \\
$\quad$ Accuracy (Percent correct in Period 4) & .22 & .03 \\
Learning & & .03 \\
$\quad$ Improvement in Accuracy (Period 3 to 4) & .22 & .03 \\
\hline
\end{tabular}

\section{Discussion}

By frequently assessing self-efficacy during learning, we were able to observe changes in self-efficacy and dynamic relations between efficacy and learning. Analyses revealed that most learners reported reliable changes in their self-efficacy during the learning task, that feelings of self-efficacy were initially associated with prior performance, and that over time students increasingly informed their self-efficacy judgments by using additional sources of information about performance such as the fluency of their problem solving. Additional analyses exploring how changes in efficacy influence future behavior, performance, and learning revealed that increases in self-efficacy were associated with less frequent help seeking, greater accuracy during problemsolving, and additional improvements in the subsequent block of problems. The results of these analyses provide confirmatory evidence and new insights that can inform theories of self-efficacy and self-regulated learning, and may have implications for educators who aim to support student learning by helping students develop efficacy for learning tasks.

Implications for self-efficacy theory

Research questions 1 and 2 tested assumptions about the nature of self-efficacy as a variable, context-specific motivational construct that is informed by perceptions of past performance. The results of our investigations into the stability and change in self-efficacy during learning confirm that self-efficacy does vary over the course of a learning task. The majority of learners $(60 \%)$ reported one or more reliable changes in their efficacy for solving math problems during the unit. These results confirm the theoretical assumption that efficacy changes during learning and should be considered a dynamic component of the self-regulated learning cycle. As such, examination of changes in efficacy during learning and their implications for future learning behavior is warranted.

As demonstrated in Fig. 2, high school math students' self-efficacy judgments were initially predicated on their performance on prior math problems. This analysis provides at least some confirmation of Bandura's $(1986,1997)$ theoretical assumption that learners base their efficacy judgments on past evidence of their ability (or lack of ability) to complete a task. In this model, the predictive path from performance to subsequent efficacy judgment diminished over time, limiting the degree to which our initial analyses confirmed Bandura's assumption. However, our later analyses (Table 4) also provide confirmatory evidence regarding the sources learners may use to inform efficacy judgments (Usher and Pajares 2006). Correlational analyses showed an increasingly strong association between measures of fluency and self-efficacy over the course of the 
learning episode. We speculate that, with fewer errors being made, accuracy of prior performance ceased to serve as a useful heuristic. To further assess their ability on the problem type, students turned to an alternative measure of their ability to complete the task: the fluency of problem solving, measured by the speed of completion of a problem step (in seconds). The idea that learners consider multiple aspects of their performance to inform efficacy judgments recasts how we think about sources of efficacy and the complexity of efficacy judgments. In the context of problem-solving with an ITS, learners seem to have considered both accuracy and fluency when making judgments and showed a flexible ability to rely on different heuristics based on the utility of information about their performance. In future studies, it may be interesting to further explore how students coordinate different sources of information about performance to make efficacy judgments (e.g., skillmeters, provide skillfulness information that can efficacy judgments, but their use is not presently logged by the software). These future studies should also be conducted with more diverse samples in order to confirm the generalizability of the findings we report.

\section{Implications for self-regulated learning theory}

The existence of variation in self-efficacy supports the assumption that self-efficacy changes over a learning task, potentially in response to other SRL processes. Results from path and correlational analyses (Fig. 3; Table 4) demonstrated that self-efficacy reports were informed by prior problem solving performance, which confirms that learners' efficacy changed in response to perceptions about the learning task. These data align to a process in which learners iteratively redefine the learning task by making comparisons between products of prior learning activities (i.e., prior accuracy and fluency) and standards (i.e., learners' feelings of efficacy for problem solving ability; Winne and Hadwin 1998, 2008) and self-reflection on motivation (Zimmerman 2011). The correlations presented in Table 5 also confirm that change in self-efficacy influences self-regulated learning behavior, performance, and learning in future SRL cycles. Thus changes in perceptions of the task (i.e., increases in efficacy over one period) prompted students to alter the metacognitive control processes they employed (i.e., a shift from help-seeking to problem-solving attempts in the next).

This investigation is the first to prompt frequent judgments of efficacy and use them to both examine how efficacy is informed by prior learning events and influences future learning. In addition to self-efficacy, SRL theory posits that multiple motivational factors operate dynamically during learning (e.g., beliefs, dispositions, orientations, Winne and Hadwin 2008, p. 299; goal orientation, interest, task value, Zimmerman and Schunk 2008, p. 7). Accordingly, similar investigations examining how achievement goals, interest, utility value, and other relevant motivational factors vary during a task and relate to learning processes would further improve our understanding of the role of motivation in learning.

\section{Implications for learning math with Cognitive Tutor Algebra}

By examining a single math unit in an intelligent tutoring system, we were able to determine that the efficacy that most high school math students feel for problem solving varies during the course of learning. Those students who experienced increases in efficacy tended to improve their learning outcomes in future periods of problem solving, which suggests that feeling efficacious about one's ability to solve math problems has positive effects on one's ability to do so, even after controlling for one's performance on prior problems. Because we know that students' efficacy judgments stem from the perception of prior achievement (Bandura 1997; Usher and Pajares 2006), it may be beneficial for educators to ensure students are aware of 
their prior successes. Providing students with this information can help them recognize growth in their skill mastery. In the context of Cognitive Tutor, interventions that improve students' interpretation of skillmeters that provide students with information on their improvements in skill mastery hold much promise (Long and Aleven 2013). By helping students recognize the development of their problem solving skills, educators can help students (accurately) increase in efficacy, which should improve their future learning outcomes.

\section{References}

Ainley, M., \& Patrick, L. (2006). Measuring self-regulated learning processes through tracking patterns of student interaction with achievement activities. Educational Psychology Review, 18(3), 267-286. doi:10. 1007/s10648-006-9018.

Aleven, V., McLaren, B., Roll, I., \& Koedinger, K. (2006). Toward meta-cognitive tutoring: a model of help seeking with a Cognitive Tutor. International Journal of Artificial Intelligence in Education, 16(2), 101-128.

Aleven, V., Roll, I., McLaren, B. M., \& Koedinger, K. R. (2010). Automated, unobtrusive, action-by-action assessment of self-regulation during learning with an intelligent tutoring system. Educational Psychologist, 45(4), 224-233.

Azevedo, R. (2005). Using hypermedia as a metacognitive tool for enhancing student learning? The role of selfregulated learning. Educational Psychologist, 40(4), 199-209.

Azevedo, R., Moos, D. C., Johnson, A. M., \& Chauncey, A. D. (2010). Measuring cognitive and metacognitive regulatory processes during hypermedia learning: issues and challenges. Educational Psychologist, 45(4), 210-223.

Azevedo, R., Harley, J., Trevors, G., Duffy, M., Feyzi-Behnagh, R., Bouchet, F., \& Landis, R. (2013). Using trace data to examine the complex roles of cognitive, metacognitive, and emotional self-regulatory processes during learning with multi-agent systems. In International Handbook of Metacognition and Learning Technologies (pp. 427-449). New York: Springer.

Bandura, A. (1986). Social foundations of thought and action: A social cognitive theory. Englewood Cliffs: Prentice-Hall.

Bandura, A. (1994). Self-efficacy. In V. S. Ramachaudran (Ed.), Encyclopedia of human behavior (Vol. 4, pp. 71-81). New York: Academic.

Bandura, A. (1997). Self-efficacy: The exercise of control. New York: Freeman.

Bandura, A. (2006). Guide for constructing self-efficacy scales. Self-efficacy Beliefs of Adolescents, 5, 307-337.

Bandura, A., \& Schunk, D. H. (1981). Cultivating competence, self-efficacy, and intrinsic interest through proximal self-motivation. Journal of Personality and Social Psychology, 41, 586-598.

Bandura, A., Reese, L., \& Adams, N. E. (1982). Microanalysis of action and fear arousal as a function of differential levels of perceived self-efficacy. Journal of Personality and Social Psychology, 43(1), 5-21.

Bernacki, M. L., Aleven, V., \& Nokes-Malach, T. J. (2014). Stability and change in adolescents' task specific achievement goals for learning mathematics with an intelligent tutoring system. Computers in Human Behavior, 37, 73-80. doi:10.1016/2014.04.009.

Bernacki, M. L., Nokes-Malach, T. J., \& Aleven, V. (2013). Fine-Grained assessment of motivation over long periods of learning with an intelligent tutoring system: Methodology, advantages, and preliminary results. In International handbook of metacognition and learning technologies (pp. 629-644). New York, NY: Springer New York. doi:10.1007/978-1-4419-5546-3_4.

Carnegie Learning. (2011). Cognitive Tutor Algebra [computer software]. Pittsburgh: Carnegie Learning, Inc.

Caspi, A., Roberts, B. W., \& Shiner, R. L. (2005). Personality development: stability and change. Annual Review of Psychology, 56, 453-484. doi:10.1146/annurev.psych.55.090902.14191.

Christensen, L., \& Mendoza, J. L. (1986). A method of assessing change in a single subject: an alteration of the RC index. Behavior Therapy, 17, 305-308.

Cleary, T. J. (2011). Emergence of self-regulated learning microanalysis: Historical overview, essential features, and implications for research and practice. In B. J. Zimmerman \& D. H. Schunk (Eds.), Handbook of selfregulation of learning and performance (pp. 329-345). New York: Routledge.

Cleary, T. J., \& Zimmerman, B. J. (2001). Self-regulation differences during athletic practice by experts, nonexperts, and novices. Journal of Applied Sport Psychology, 13(2), 185-206. 
Efklides, A. (2011). Interactions of metacognition with motivation and affect in self-regulated learning: the MASRL model. Educational Psychologist, 46(1), 6-25. doi:10.1080/00461520.2011.53864.

Ericsson, K. A., \& Simon, H. A. (1980). Verbal reports as data. Psychological Review, 87(3), 215-251.

Fryer, J. W., \& Elliot, A. J. (2007). Stability and change in achievement goals. Journal of Educational Psychology, 99(4), 700-714.

Greene, J. A., \& Azevedo, R. (2007). Adolescents' use of self-regulatory processes and their relation to qualitative mental model shifts while using hypermedia. Journal of Educational Computing Research, $36(2), 125-148$.

Greene, J. A., \& Azevedo, R. (2009). A macro-level analysis of SRL processes and their relations to the acquisition of a sophisticated mental model of a complex system. Contemporary Educational Psychology, 34(1), 18-29. doi:10.1016/j.cedpsych.2008.05.00.

Greene, J. A., Robertson, J., \& Costa, L. J. C. (2011). Assessing self-regulated learning using think-aloud methods. In B. J. Zimmerman \& D. H. Schunk (Eds.), Handbook of self-regulation of learning and performance (pp. 313-328). New York: Routledge.

Hackett, G., \& Betz, N. E. (1989). An exploration of the mathematics self-efficacy/mathematics performance correspondence. Journal for Research in Mathematics Education, 20, 263-271.

Jacobson, N. S., \& Truax, P. (1991). Clinical significance: a statistical approach to defining meaningful change in psychotherapy research. Journal of Consulting and Clinical Psychology, 59(1), 12.

Koedinger, K. R., \& Aleven, V. (2007). Exploring the assistance dilemma in experiments with cognitive tutors. Educational Psychology Review, 19(3), 239-264. doi:10.1007/s10648-007-9049.

Koedinger, K. R., \& Corbett, A. (2006). Cognitive tutors: Technology bridging learning sciences to the classroom. In R. K. Sawyer (Ed.), Cambridge handbook of the learning sciences (pp. 61-78). New York: Cambridge University Press.

Lent, R. W., Brown, S. D., \& Larking, K. C. (1984). Relation of self-efficacy expectations to academic achievement and persistence. Journal of Counseling Psychology, 31, 356-362.

Long, Y., \& Aleven, V. (2013). Supporting students' self-regulated learning with an open learner model in a linear equation tutor. In H. C. Lane, K. Yacef, J. Mostow, \& P. Pavlik (Eds.), Proceedings of the 16th International Conference on Artificial Intelligence in Education (AIED 2013) (pp. 249-258). Berlin: Springer.

McQuiggan, S. W., Mott, B. W., \& Lester, J. C. (2008). Modeling self-efficacy in intelligent tutoring systems: an inductive approach. User Modeling and User-Adapted Interaction, 18(1), 81-123.

Moos, D. C. (2014). Setting the stage for the metacognition during hypermedia learning: what motivation constructs matter? Computers \& Education, 70, 128-137. doi:10.1016/j.compedu.2013.08.01.

Muis, K. R., \& Edwards, O. (2009). Examining the stability of achievement goal orientation. Contemporary Educational Psychology, 34, 265-277.

Pajares, F. (2008). Motivational role of self-efficacy belies in self-regulated learning. In D. H. Schunk \& B. J. Zimmerman (Eds.), Motivation and self-regulated learning: Theory, research, and applications. New York: Erlbaum.

Pajares, F., \& Miller, M. D. (1994). Role of self-efficacy and self-concept beliefs in mathematical problem solving: a path analysis. Journal of Educational Psychology, 86, 193-203.

Pintrich, P. R. (2000). The role of goal orientation in self-regulated learning. In B. Pintrich \& Zeidner (Eds.), Handbook of self-regulation (pp. 451-502). San Diego: Academic Press.

Pintrich, P.R., Smith, D.A.F., Garcia, T., \& McKeachie, W.J. (1991). A manual for the use of the motivated strategies for learning questionnaire (MSLQ) (tech. Rep. No. 91-B-004). Ann Arbor: University of Michigan, School of Education.

Rosenthal, R., \& Rosnow, R. L. (1991). Essentials of behavioral research: Methods and data analysis (Vol. 2). New York: McGraw-Hill.

Shell, D. F., \& Husman, J. (2008). Control, motivation, affect, and strategic self-regulation in the college classroom: a multidimensional phenomenon. Journal of Educational Psychology, 100, 443-459.

Siegler, R. S., \& Crowley, K. (1991). The microgenetic method: a direct means for studying cognitive development. American Psychologist, 46, 606-620.

Usher, E. L., \& Pajares, F. (2006). Sources of academic and self-regulatory efficacy beliefs of entering middle school students. Contemporary Educational Psychology, 31(2), 125-141. doi:10.1016/j.cedpsych.2005.03.00.

Winne, P. H. (2011). A cognitive and metacognitive analysis of self-regulated learning. In B. J. Zimmerman \& D. H. Schunk (Eds.), Handbook of self-regulation of learning and performance (pp. 15-32). New York: Routledge.

Winne, P. H., \& Hadwin, A. F. (1998). Studying as self-regulated learning. In D. J. Hacker, J. Dunlosky, A. C. Graesser, D. J. Hacker, J. Dunlosky, \& A. C. Graesser (Eds.), Metacognition in educational theory and practice (pp. 277-304). Mahwah: Lawrence Erlbaum Associates Publishers.

Winne, P. H., \& Hadwin, A. F. (2008). The weave of motivation and self-regulated learning. In D. H. Schunk \& B. J. Zimmerman (Eds.), Motivation and self-regulated learning: Theory, research, and applications (pp. 297-314). Mahwah: Erlbaum. 
Winne, P. H., \& Perry, N. E. (2000). Measuring self-regulated learning. In M. Boekaerts, P. R. Pintrich, \& M. Zeidner (Eds.), Handbook of self-regulation (pp. 531-566). San Diego: Academic.

Zimmerman, B. J. (2000a). Attaining self-regulation: A social cognitive perspective. In M. Boekaerts, P. R. Pintrich, \& M. Zeidner (Eds.), Handbook of self-regulation (pp. 13-39). San Diego: Academic.

Zimmerman, B. J. (2000b). Self-efficacy: an essential motive to learn. Contemporary Educational Psychology, 25(1), 82-91. doi:10.1006/ceps.1999.101.

Zimmerman, B. J. (2011). Motivational sources and outcomes of self-regulated learning and performance. In B. J. Zimmerman \& D. H. Schunk (Eds.), Handbook of self-regulation of learning and performance (pp. 4964). New York: Routledge.

Zimmerman, B. J., \& Schunk, D. H. (2008). Motivation: An essential dimension of self-regulated learning. In D. H. Schunk \& B. J. Zimmerman (Eds.), Motivation and self-regulated learning: Theory, research, and applications (pp. 1-30). New York: Routledge.

Zimmerman, B. J., \& Schunk, D. H. (Eds.). (2011). Handbook of self-regulation of learning and performance (pp. 49-64). New York: Routledge. 